\title{
Chatting through Pictures? A Classification of Images Tweeted in one week in the UK and USA ${ }^{1}$
}

Mike Thelwall, Statistical Cybermetrics Research Group, University of Wolverhampton. Olga Goriunova, Centre for Interdisciplinary Methodologies, University of Warwick. Farida Vis, Information School, University of Sheffield.

Simon Faulkner, Manchester School of Art, Manchester Metropolitan University. Anne Burns, Information School, University of Sheffield.

Jim Aulich, Manchester School of Art, Manchester Metropolitan University.

Amalia Mas-Bleda, Institute of Public Goods and Policies, Spanish National Research Council. Emma Stuart, Statistical Cybermetrics Research Group, University of Wolverhampton.

Francesco D'Orazio. Pulsar, London.

Twitter is used by a substantial minority of the populations of many countries to share short messages, sometimes including images. Nevertheless, despite some research into specific images, such as selfies, and a few news stories about specific tweeted photographs, little is known about the types of images that are routinely shared. In response, this article reports a content analysis of random samples of 800 images tweeted from the UK or USA during a week at the end of 2014. Although most images were photographs, a substantial minority were hybrid or layered image forms: phone screenshots, collages, captioned pictures, and pictures of text messages. About half were primarily of one or more people, including $10 \%$ that were selfies, but a wide variety of other things were also pictured. Some of the images were for advertising or to share a joke but in most cases the purpose of the tweet seemed to be to share the minutiae of daily lives, performing the function of chat or gossip, sometimes in innovative ways.

\section{Introduction}

Sharing images through social media is common in richer nations. In 2012, $45 \%$ of adult internet users in the USA had posted their own photographs online $67 \%$ of those aged 18 29) and $35 \%$ had shared photographs created by others (52\% of those aged 18-29) (Rainie, Brenner, \& Purcell, 2012). In the USA in 2013, 17\% of adults on the internet used Twitter and $71 \%$ used Facebook, both of which can be used to share pictures, and $16 \%$ used the image sharing site Instagram (Duggan \& Smith, 2013). In the UK in 2013, 70\% of adults involved in online activities reported sharing images (Dutton, Blank, \& Groselj, 2013) and it seems likely that by the end of 2014 the majority of people using internet in both countries had shared images online. Images are particularly likely to get retweeted in Twitter, and hence seem to be an important component of its ecosystem (Rogers, 2014). These statistics, however, do not reveal anything about what types of images are shared and why they are shared.

Press coverage of Twitter sometimes focuses on celebrity users or on public tweets in reaction to major events and news stories. Although there are instances in which the role of images in these activities drew a significant amount of attention (Vis et al., 2013),

\footnotetext{
${ }^{1}$ This is a preprint of an article published in the Journal of the Association for Information Science and Technology (C) copyright 2015 John Wiley \& Sons, Inc. Thelwall, M., Goriunova, O. Vis, F., Faulkner, S., Burns, A., Aulich, J. Mas-Bleda, A., Stuart, E. \& D'Orazio, F. (2016). Chatting through pictures? A classification of images tweeted in one week in the UK and USA. Journal of the Association for Information Science and Technology, 67(11), 2575-2586. doi:10.1002/asi.23620
} 
research projects dedicated to high-profile cases, such as misogynistic abuse on Twitter of Criado-Perez and other women campaigners for a woman to appear on a UK banknote do not necessarily focus on the role and nature of images in these events. While tweeting is a convenient way to share more traditional family or party photography, such everyday image-sharing seems to be overlooked in comparison to the high profile uses of Twitter. This is a serious omission because without this context it is impossible to fully evaluate the significance of the adaptations of visual culture within social media and the birth of the new phenomena that do get noticed, such as photobombing. This context will also inform debates about how image-sharing practices feed into commercial and socio-political uses of images on Twitter.

In response to the lack of general information about the types of images typically shared on social media, this article reports a content analysis of random samples of images tweeted in the USA and UK. Twitter was chosen as one of the most common social media services and one that is frequently used for image sharing. Twitter is also used to share images originally posted in other sites, such as Instagram, Pinterest, Flickr or Tumblr, giving it a central role in the image-sharing ecosystem. The UK and USA were chosen as they are amongst the biggest Twitter users $\left(1^{\text {st }}\right.$ and $4^{\text {th }}$, according to Alexa.com in February, 2015) and are relatively similar in terms of shared languages and culture, allowing an interesting comparison.

\section{Social uses of images}

Most Twitter users tweet in a personal capacity, although journalists and media bloggers being also relatively common, and corporate accounts form a small minority (De Choudhury, Diakopoulos, \& Naaman, 2012). Thus, personal use of Twitter may dominate image dissemination. There is no specific evidence to justify this claim, however and, since most URLs shared in Twitter originate from a small number of elite media bloggers (Wu, Hofman, Mason, \& Watts, 2011), it is possible that most images shared are also taken by an elite minority.

Information about how people tweet can help to understand the context of the pictures that they tweet. A content analysis of up to ten tweets each from 350 randomly selected active personal Twitter users (excluding corporate and self-promoting users) found that the most common types of tweets were the (overlapping) categories: me now $(40 \%)$, statements/random thoughts (23\%), opinions/complaints (23\%) and information sharing (22\%) (Naaman, Boase, \& Lai, 2010). This suggests that whilst most users ("meformers") primarily share personal information, another large group post more general information. If this extends to images then there could be many images of a personal nature in addition to images with a more general context. Hence, typical images in social media seem likely to be personal or for some type of information sharing.

\section{Personal image and photo sharing (meformers)}

The widespread uptake of photography (Braden, 1983; Beegan, 2008; Benjamin, 1936; Marien, 2014) has allowed it to be embedded in people's lives through everyday family photographs (Rose, 2010) and for events, such as weddings and holidays, where the visual is important for long term memories (Berger, 2013; Cobley \& Haeffner, 2009; Urry \& Larsen, 2011; Robinson \& Picard, 2009). The internet has given individuals potential access to large audiences for their images and smartphones have allowed them to take and distribute large numbers of photographs of all aspects of their lives. For example, Twitter allows users to send images directly via their smartphone, via SMS (Twitter Blog, 2011a), through Apple and 
other third party smartphone apps (Twitter Blog, 2011b) or their computer (Taylor, 2011; Twitter Help Centre, no date).

The advent of digital technology has triggered new uses for photography (Miller \& Edwards, 2007; Van House, Davis, Ames, Finn, \& Viswanathan, 2005; Gomez, Cruz \& Meyer, 2012). Photo-sharing in social media is not a simple extension of its offline predecessors, however. For example, existing traditions of displaying photographs of the dead (Brown, 2013), have become more prominent and less socially compartmentalised in social media (Cann, 2014). Social media-specific sharing practices are also evident in a range of new image genres such as the selfie, photobomb and duckface.

The extent to which images communicate in a way comparable to verbal language is unclear because it 'is not clear that they actually "say" anything' (Mitchell, 2005: 140), although in social media they are used to express strong positive sentiments (Bourlai \& Herring, 2014). Photographic images look like pieces of the world as much as statements about it (Sontag, 1979), but chat can nevertheless occur alongside the images and incorporates them into its primarily verbal discourse (Hu, Manikonda, \& Kambhampati, 2014). Offline experiments have also shown that photograph sharing can trigger discussions and introduce novel topics that would not otherwise be discussed and so they seem to have a valuable role in communication (ten Bhömer, Helmes, O'Hara, \& van den Hoven, 2010). Moreover, the instant sharing of images of everyday life can create a sense of remote presence with friends, helping to sustain relationships and interact with friends at a distance (Ibrahim, 2015). This can explain the apparent prevalence of apparently trivial images within social media (e.g., Hu, Manikonda, Kambhampati, 2014). Nevertheless, sharing photographs online can be socially important for the sharer, and particularly for teens, who may carefully select apparently trivial images to project a desired identity or performance to their friends (Durrant, Frohlich, Sellen, \& Uzzell, 2011; Van Dijck, 2008; Zhao, Salehi, Naranjit, Alwaalan, Voida, \& Cosley, 2013). In addition, photograph sharing can be an important aspect of communication between friends (Van House, 2011). Finding out about friends and acquaintances is one of the key functions of gossip, which is an important information gathering activity (Dunbar, 1998) and a key reason for the success of social network sites (Donath, 2007; Tufekci, 2008).

\section{Information Sharing (informers): News, Celebrities and memes}

News sharing is an important activity in Twitter (Java, Song, Finin, \& Tseng, 2007) and so news-related images may be expected in any large sample, perhaps from media and journalist sources rather than individually taken. Nevertheless, individual social media images have had a significant impact on the news. A selfie from Amanda Knox, an American woman accused of murder in Italy, has been credited as being influential in her campaign (Clifford, 2014), phones have given access to real-time images of crises (Reading, 2011), for news where journalists are not present (Chung \& Yoon, 2013) and first hand evidence about ongoing conflicts from photojournalists (Alper, 2014) and participants on all sides (Klausen, in press). Fake news images are also common (Burgess, Vis \& Bruns, 2012; Gupta, Lamba \& Kumaraguru, 2013; Gupta, Lamba, Kumaraguru \& Joshi, 2013).

Social media profiles are increasingly seen as important for politicians to get their messages across (Broersma \& Graham, 2012; Ekman \& Widholm, 2014; Parmelee \& Bichard, 2011). Images may not be an important part of political tweeting, however, although there are exceptions (BBC, 2014).

Celebrities are influential on Twitter (De Choudhury, Diakopoulos, \& Naaman, 2012; Kassing \& Sanderson, 2010; Sanderson \& Cheong, 2010). The importance of celebrities for Twitter is such that interest in them has been shown to associate with uptake of the service 
amongst young US adults (Hargittai \& Litt, 2011). Following tweets can give an impression of direct real-time access to the celebrity users (Click, Lee, \& Holladay, 2013), particularly if they tweet images from their smartphones (Marwick \& boyd, 2011), such as selfies (Collings, 2014), and so retweets of celebrity images may be common in Twitter.

Other common topics of news-related conversation, such as sport (Hutchins, 2011; Kassing \& Sanderson, 2010), are also likely to be represented in Twitter. Presumably, more photogenic activities are more likely to be represented by shared images.

Joke sharing has been important within many online cultures, particularly in the form of memes (Levy, 2001; Goriunova, 2012, 2014; Ensmenger, 2010; Shifman, 2013). An internet meme is often an image or a family of images that generates a culture of re-makes, virally spreads beyond its original subculture, and is native to the internet (Goriunova, 2013). A popular type of meme is an image overlaid with a sans-serif font text (i.e., an image macro). Lolcats, for example, are unusual photographs of cats with deliberately ungrammatical captions (Brubaker, 2008; Leigh, 2009; Miltner, 2011). Many memes are based on customised pictures, whether photographs or animations, and seem to be generated for comedy or sarcasm (Wiggins \& Bowers, 2014). The use of text within or associated with images continues a tradition within painting (Alpers, 1983; van Straten, 1994) and photography (Hunter, 1987; Mitchell 1994).

Twitter is also widely used for sharing marketing information (Thoring, 2011; Wood \& Burkhalter, 2014), presumably with frequent images of products for sale.

\section{Analyses of social media images}

Despite the many years of image sharing on social media, there is surprisingly little published academic research that has focused on the images themselves. This is in contrast to extensive research about fine art images (Panofsky, 1983), photographers and photographs (Marien, 2014), and snapshot photography (Chalfen, 1987; Holland \& Spence, 1991; Sarvas \& Frohlich, 2011; Larsen \& Sandbye, 2014; Batchen, 2008; Gomez Cruz \& Ardévol, 2013). A content analysis of a random sample of images, with any associated text, shared on Tumblr and associated with one of five highly popular fan community hashtags (e.g., \#onedirection) or one general tag (\#feels) found that they contained more and stronger sentiment in comparison to similar image-free posts. Moreover, posts with images tended to contain positive emotions $(57 \%)$ whereas equivalent posts without images tended to convey negative emotions (68\%). One possible reason for the difference is that users wishing to let out negative feelings (sometimes signalled with \#vent or \#rant) tend not to take the time to find an appropriate image (Bourlai \& Herring, 2014). A content analysis of 109 images shared by 40 users recruited from the USA and Taiwan from a custom-built instant messaging mobile phone app found that the images were shared as part of ongoing communications rather than as one-off entities in their own right. Screenshots were also singled out in this context as a device that allowed complex messages to be communicated accurately, such as reservation details, without extensive typing (Chen, Bentley, Holz, \& Xu, in press).

One study has focused more generally on the content of social media images. It used a sample of 50 regular active Instagram users and manually coded their most recently posted 20 pictures each. Images were automatically sorted into 15 visually similar groups, with subsequent human adjustments and mergers to make a final set of 8 groups: Pet (3\%); Fashion (4\%); Food (10\%); Gadget (11\%); Captioned photo (11\%); Activities (15\%); Friends (22\%); Selfies (24\%) (Hu, Manikonda, Kambhampati, 2014). 


\section{Research Questions}

This is an exploratory, data-driven study about the types of images shared on Twitter. For this study, an image is anything within an electronic image file format, even if the image is blank or is of text alone. The goal is to identify the main types of images that are tweeted, giving background information about typical image making and sharing as well as insights into the aspects of everyday Twitter images that need to be further researched. The latter goal is an information-centred approach (Thelwall, Wouters, \& Fry, 2008). In consequence, the main research questions are very general.

1. Which types of images are shared on Twitter?

2. What are images shared on Twitter of?

3. Why are these images shared on Twitter?

4. When are images shared on Twitter?

The study also assesses, as a fifth research question, whether there are substantial differences between the UK and the USA in the answers to the above questions. The purpose of this is to get insights into whether there are likely to be major differences in Twitter use between communities. Presumably two very different cultures would have very different practices and purposes for image sharing but it is less obvious that the UK and USA would have substantially different uses.

\section{Methods}

Tweets were first sampled from the UK and USA using the free software Webometric Analyst to query the public Twitter Applications Programming Interface (API) over exactly 7 days from November 29, 2014 at 12.43 GMT. In each case a blank query was used in conjunction with a geographic restriction consisting of a large circle on the earth encompassing a majority of the country, although it also catches small parts of neighbouring countries: Mexico for the USA (as well as many Spanish-speaking residents of the USA) and Ireland for the UK. This method should retrieve a sample of under $1 \%$ of relevant tweets, a Twitter API restriction. The data collection produced:

- $1,876,484$ UK tweets, 364,802 with URLs, and 196,600 pictures were downloaded

- 1,484,474 USA tweets, 292,172 with URLs, and 133,096 pictures were downloaded

Each tweet was processed to extract and resolve any links (i.e., following server redirects from image shortening services and other sources). Resolved link URLs ending in .png, jpg, jpeg or .gif were judged to be image URLs and were downloaded and saved. Thus, only natively shared images and hyperlinks to image files were downloaded and not images that were originally posted elsewhere and tweeted in the form of a link to the containing webpage rather than the image file itself. A random sample of 400 images was then extracted by Webometric Analyst for each country and used for the content analysis. The sampling procedure used a random number generator to select from the set of downloaded images. The sample should reveal typical patterns of use of Twitter in the two countries, although it will be influenced by the time of year. Since the sampling is by image rather than user, the results reflect typical images rather than typical users' images, in the sense that people that tweet more images are more likely to have images selected for the set.

Content analysis is an appropriate method for analysing images because, although it is not capable of dealing with the nuances of individual images, it is able to characterise properties of a large set of images in a systematic way, avoiding at least some of the potential biases of more detailed investigations into small sets of images (Rose, 2012). Previous content analyses of images in social media have used classifications for the text associated with them, such as their tags (Angus, Thelwall, \& Stuart, 2008; see also: Bar-Ilan, 
Shoham, Idan, Miller, \& Shachak, 2008). Although these tags are important for helping users to find images (Jörgensen, 2003; Marlow, Naaman, Boyd, \& Davis, 2006), classification schemes for image text are not necessarily able to characterise the most interesting social properties of images. There is also a body of theory concerning the classification of the images themselves for cataloguing (Shatford, 1986; 1994) or information retrieval (Jörgensen, 1998) but these also focus on why people may want to find the images rather than on describing the general properties of a collection of images. However, while the classification scheme used here was informed by previous schemes, it is substantially different.

The classification scheme was built inductively by the first author by looking at the images and identifying common themes in their appearance and subjects. The classification scheme had four main facets: the overall type or format of the image (the technical angle); its subject or content (see the similar Instagram categories of: Hu, Manikonda, \& Kambhampati, 2014, and including selfies); the apparent reason for sharing it (including memetic, and celebrity); and when it was shared. The format of an image is relevant from a visual culture perspective because this signifies how it was constructed and its genre. The subject or content category is essentially Panofksy's "of" concept (Panofsky, 1983; Angus, Thelwall, \& Stuart 2008), describing from a relatively naïve perspective what an image depicts rather than attempting to interpret a deeper meaning or deeper context. This was chosen in preference to a more contextualised classification informed by the background literature in order to allow a more transparent visual analysis, although the discussion relates the results to the background literature. For the purpose category, it is not possible to be sure why an image was shared without asking the sharer but in two cases the purpose seemed obvious (advertising and jokes) and in many other cases the picture seemed to be shared to show that a person was somewhere or doing something - a bit like a personalised holiday postcard, but rarely from a holiday. The apparent time of posting of an image is relevant because Twitter is supposed to be a real time information sharing medium and so any evidence that it deviates from this may be significant in terms of understanding the culture of image sharing.

Each image was classified into just one category in each of these facets, except the second, for which a secondary category was also allowed because multiple categories were possible for some images. For example, a person holding a beer prominently to the camera represents both the person and the beer. In addition, a range of additional properties of the images were observed that were either common or of sufficient interest to identify separately (e.g., if the image was a selfie or if it contained text) and these were also recorded for each image. The images were classified in conjunction with the text of the associated tweet because images alone can be highly ambiguous (Hunter, 1987). When necessary, the Twitter profile of the originator was visited for additional context. For example, some of the pictures containing bare flesh were investigated to check whether they were produced by a sex worker and hence should be classified as commercial advertising.

Image type or format facet: One to be selected plus any of the extra categories that apply.

- Just photograph. Can have solid black bars at the top or side; can have a tiny logo or credit line.

- Mainly photograph or collage of photographs.

- Image of message(s) only or messages as the main purpose of a screen grab.

- Comic or cartoon.

- Other.

- [extra] Image contains all or part of a screen grab. 
- [extra] Image of text or image contains text that is important to the picture (not photographer, credit, URL or Twitter handle).

- [extra] Professional image or content - apparently taken by a professional photographer (not amateur pictures taken for commercial reasons) or made by a professional graphic designer.

Image content facet: One main category to be selected and one optional minor category, plus any of the extra categories that apply.

- Person or parts of person.

- Small group of people, probably 2-10 people that are easily individually identifiable.

- Large group of people, probably $11+$ people that are not easily individually identifiable or it is clear that the group is photographed rather than the individual members (e.g., from the back, from a distance).

- Animal(s).

- Food or drink (including packaged in supermarket) without a human being prominently in the picture.

- Place, such as a stadium, field, house, street or town but not a room inside a house.

- Other things, including messages, song playing on phone.

- [extra] Selfie - photo taken by one of the people in it even if others or other things are also prominently in it, not if just a part of the person's body.

- [extra] TV - is mainly a picture of a TV or a TV screen or computer used as a TV.

- [extra] Pornographic.

Image purpose facet: One to be selected plus any of the extra categories that apply.

- Advertising a product or service for sale, including restaurants or cafes.

- Joke image. The joke must be inside the picture, not the tweet text.

- Event presence - recording presence at a public event, performance, sport, meeting or similar.

- Other purpose or purpose not clear.

- [extra] Relating in some way to a famous person other than a cartoon character, or the artist of any song playing.

- [extra] Meme picture or relating clearly to a memetic use of an image or memetic image type.

- [extra] Christmas-related.

Image time relationship facet: One to be selected.

- Real-time photograph, screen grab or artwork that appears to have been just taken or created.

- Current or topical - not real-time but relating to something recent or ongoing - such as advertising for a product, sale or show (unless on a poster that has just been photographed).

- Timeless, including cartoons, memes, and emotional text messages and sayings.

- Old - the picture is clearly old or posted for historical reasons (e.g., last year, when we were young).

- Other.

The Person or parts of person category is a merger of two original categories that were difficult to separate because some pictures showed most of a person but focused on one part of them (e.g., "when I had a bigger bum" or a photo of an out of focus man holding a beer to the camera), or just showed the person's face (half a face in one case). A few photographs clearly just showed a part of a person, however, such as a hand with a ring, bandage or watch. 
To check the clarity of the classification scheme and the accuracy of the coding, two further people (both with a PhD in information science) were given the same set of images to code. A code book was drawn up that contained the above facet descriptions and technical details about how to access the pictures and enter the codes. In addition, the codes of the first author for 100 UK and 100 US pictures were given to serve as a guide to the classification scheme. Cohen's kappa inter-coder agreement rates (Cohen, 1960) were then calculated between all three coders for 597 images (excluding 200 used for training and three images that were difficult to access). For the type or format facet, only the primary code was analysed. The first author's judgment was used to resolve any disagreements.

There is not an agreed set of values for adequate agreement rates but two scales are commonly used. Fleiss (1981) describes 0.40 - as poor, $0.40-0.75$ as fair to good, and $0.75+$ as excellent. Landis and Koch (1977) characterise 0-0.20 as slight agreement, 0.21-0.40 as fair agreement, $0.41-0.60$ as moderate agreement, 0.61-0.80 as substantial agreement, and $0.81+$ as almost perfect agreement. From these, it seems reasonable to use 0.4 as the cutoff point below which the agreement level is too low to be useful. Although two of the values for the meme facet are below 0.4 , the third value for this facet is substantially above 0.4. In this case, coder $C$ seemed to be using a different interpretation of the term meme from the other two coders and so it seems reasonable to accept the codes based on the agreement of 0.551 between A and B. In the TV category, one of the three agreement rates was below the threshold of 0.4 but the other two were above it. This category was perhaps subjective because it was often not clear whether a photograph was of a TV or a computer monitor, or a screen grab. The Professional category was also quite subjective. The results from these three categories should therefore be interpreted with a degree of caution. In order to report more detailed findings, similar categories were not merged in an attempt to get higher inter-coder agreement rates.

Table 1. Cohen's kappa values for inter-coder agreement for the first author (A) and the other two coders ( $B$ and $C$ ).

\begin{tabular}{|l|l|l|l|}
\hline Facet & A vs. B & A vs. C & B vs. C \\
\hline Type or format & 0.719 & 0.691 & 0.615 \\
\hline Content & 0.821 & 0.816 & 0.805 \\
\hline Purpose & 0.605 & 0.541 & 0.485 \\
\hline Time relationship & 0.571 & 0.410 & 0.357 \\
\hline -Screen grab & 0.940 & 0.843 & 0.870 \\
\hline -Text & 0.786 & 0.796 & 0.833 \\
\hline -Professional & 0.546 & 0.491 & 0.453 \\
\hline -Selfie & 0.839 & 0.894 & 0.870 \\
\hline -TV & 0.596 & 0.335 & 0.424 \\
\hline -Pornographic & 0.940 & 0.702 & 0.747 \\
\hline -Famous & 0.639 & 0.458 & 0.495 \\
\hline -Meme & 0.551 & 0.043 & 0.046 \\
\hline - Christmas & 0.716 & 0.691 & 0.770 \\
\hline
\end{tabular}

\section{Results}

Most of the images were photographs and about two thirds were either photographs or derived from photographs (Figure 1). About $9 \%$ of the images mainly displayed text, such as chat dialogs or sayings, and about $17 \%$ were more complex constructions. The vast majority 
of the images did not seem to be professional and about $15 \%$ were screen grabs of phones presumably from people sharing what they were currently doing with their phone (e.g., listening to music, playing a game, reading a text message, checking Facebook or Twitter, using Whatsapp or checking the weather).

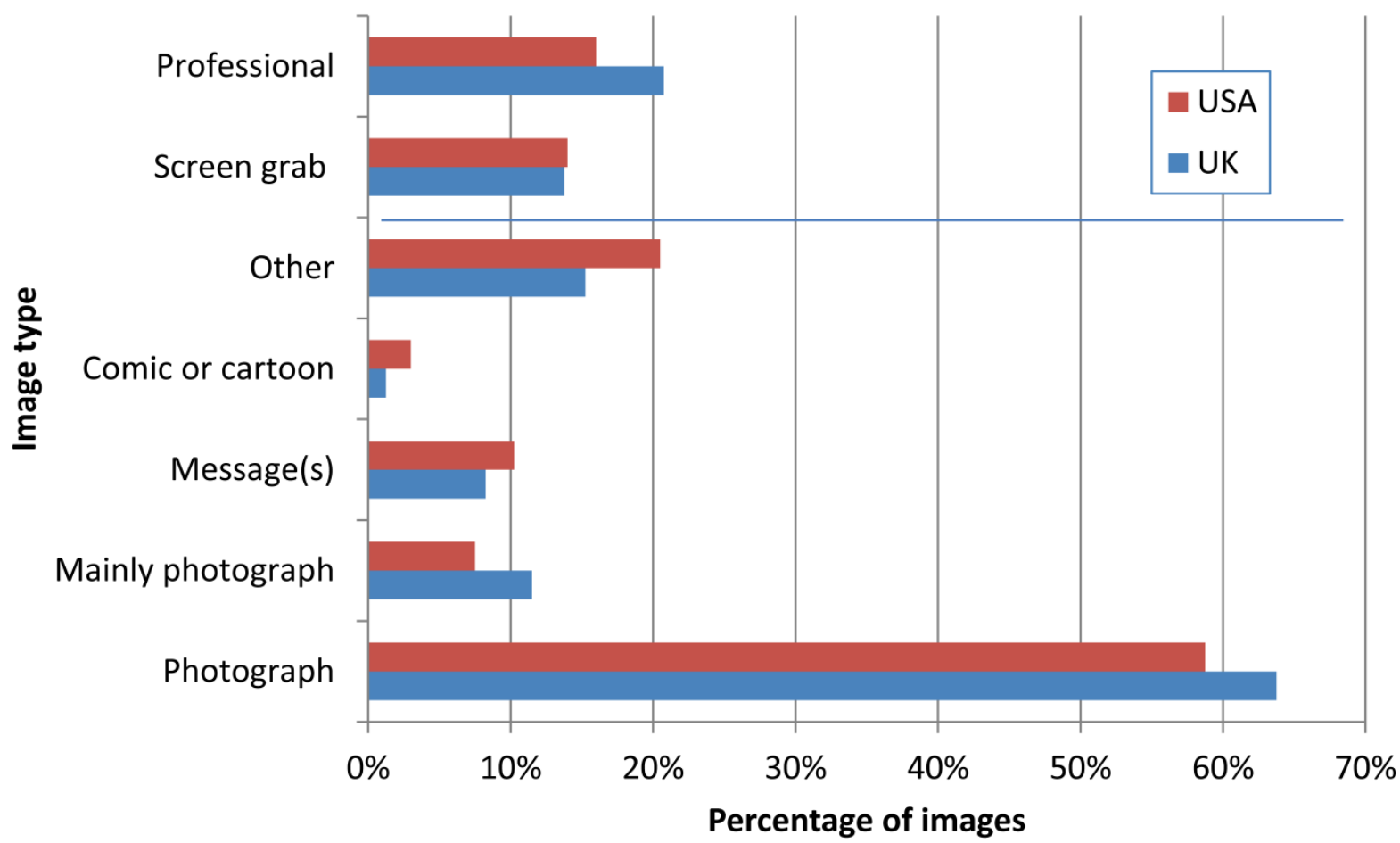

Figure 1. Random US and UK Twitter images categorised by type. The two top categories are non-exclusive.

Just under a quarter of the pictures were mainly of an individual person, with an additional $17 \%$ being of small groups, often just consisting of two people (Figure 2). Almost half of the pictures fell in the Other category - a fifth of these were pictures of text but most were of a variety of objects, such as Christmas trees. Just under a third of the pictures were either images of text or contained text as an important component, such as an overlaid caption. 


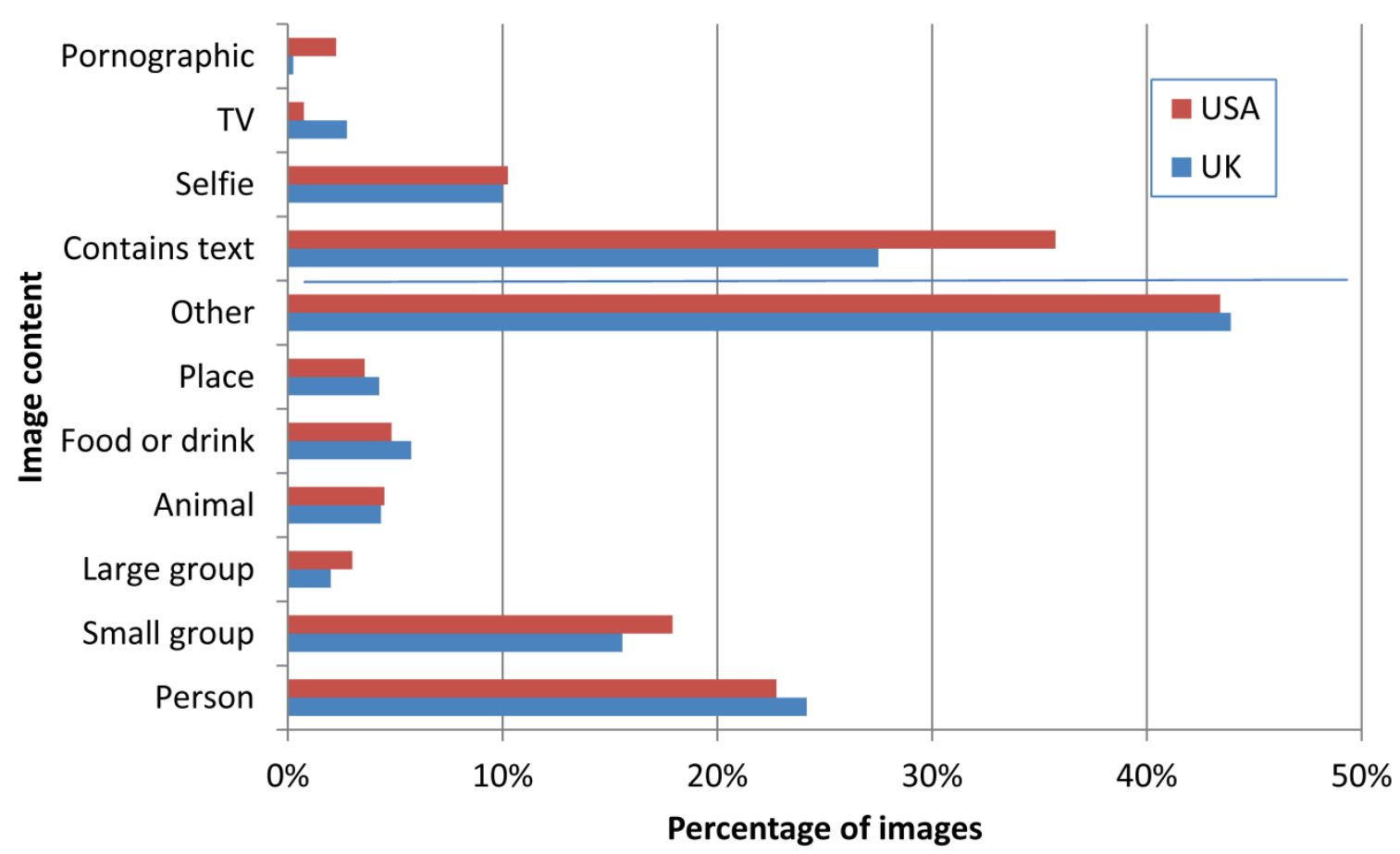

Figure 2. Random US and UK Twitter images categorised by content. The four top categories are non-exclusive. The remaining categories are normalised when two were selected for an image: the main category was weighted at $2 / 3$ and the secondary category at $1 / 3$ in order to make the primary category twice as important as the secondary category - the simplest reasonable combination.

Few tweets revealed a clear reason for posting an image, although some pictures were advertisements or advertised a product or service (Figure 3). As an example of the Other category, several pictures photographed Christmas decorations, perhaps to show how they looked or to announce that the decorations had just been put up. About $15 \%$ of the images were of famous people or related to them in some way. Few pictures were clearly identified as either a joke or a meme.

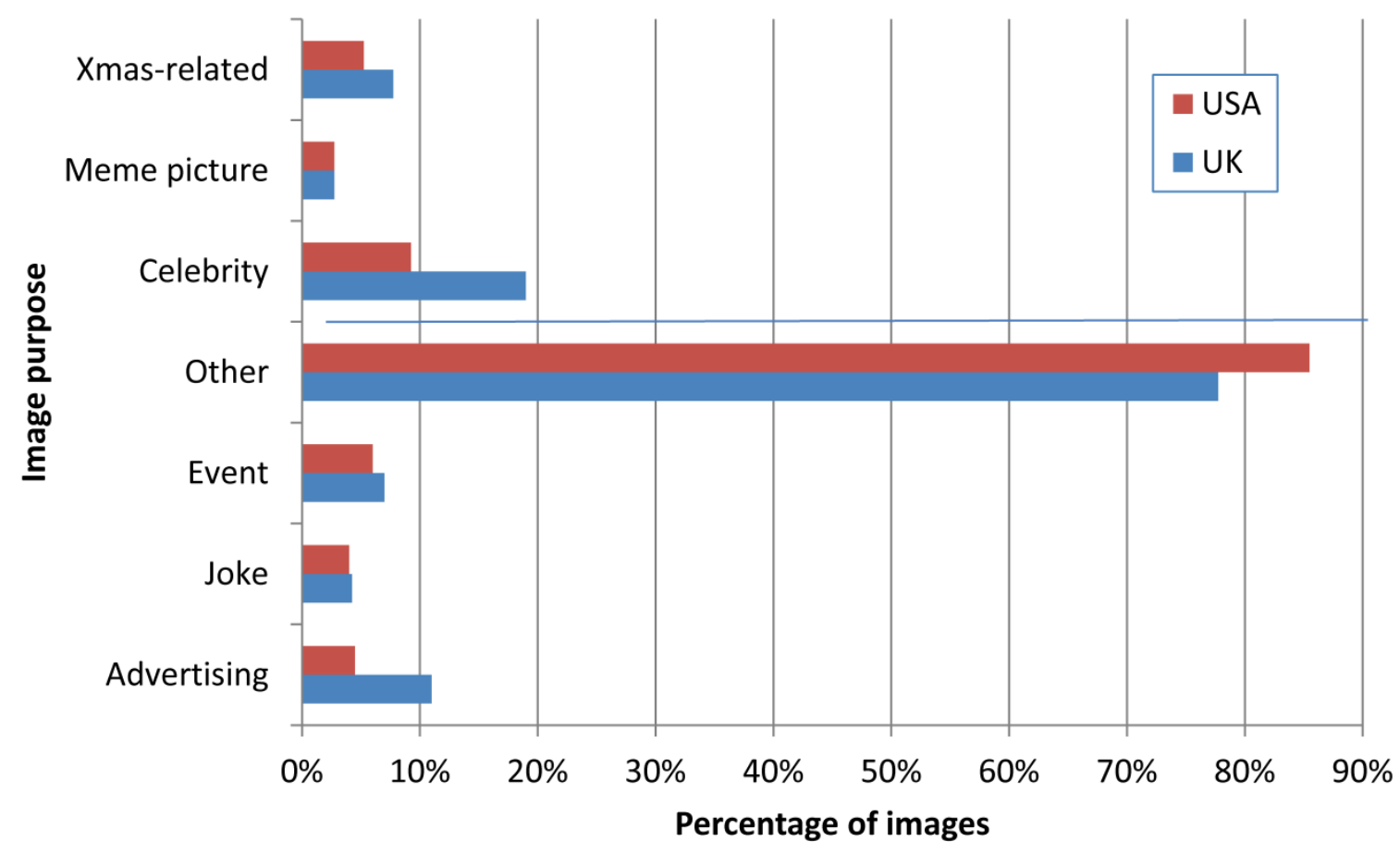


Figure 3. Random US and UK Twitter images categorised by apparent purpose. The three top categories are non-exclusive.

About two thirds of the images seemed to have been just created (Figure 4), although this high figure was partly due to the assumption that a picture shared without any time context in the associated tweet would have been recently taken, which may not always have been true.

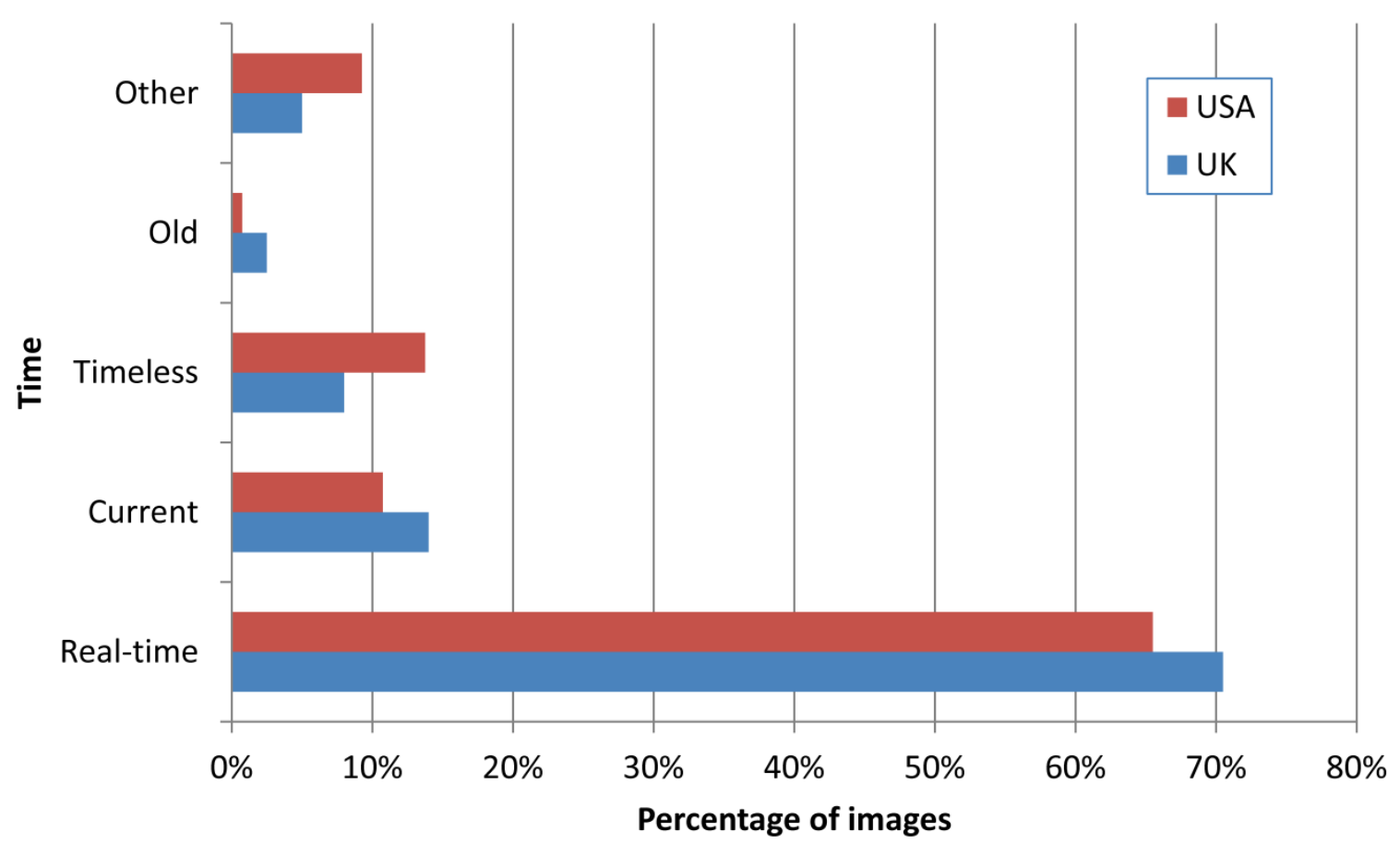

Figure 4. Random US and UK Twitter images categorised by time orientation.

The Other categories are quite large for a content analysis, especially for the image purpose facet. This is due to a combination of the wide variety of reasons why Twitter is used for image sharing and an attempt at a relatively specific classification scheme.

\section{Analysis of emergent themes}

This section discuses aspects of the results that seem to be of particular interest and relates some to the meformer/informer dichotomy introduced above. Two thirds of the images in this study are photographs or derivatives, and so digital photography is clearly important. The photographs shared primarily depict current content, although perceptions of what is current are affected by the system used (Weltevrede, Helmond \& Gerlitz, 2014). Selfies formed $10 \%$ of the images and are a meformer strategy. Most $(56 \%)$ were individual selfies and others were selfies with a friend or a few others (42\%). In all except three (91\%) of the selfies with more than one person, at least one of the people was smiling, looked amused, or looked happy - often very happy. In contrast, only $44 \%$ of the people in solo selfies looked happy in any way, often looking serious or focused on taking the selfie instead. Most selfies did not contain anything prominent except people, although a few also featured a pet $(4 \%)$, place $(5 \%)$, food $(3 \%)$ or something else $(4 \%)$. Selfies were sometimes taken at arm's length, sometimes in a mirror and at least one was taken with a selfie stick. Eight of the selfies contained the duckface pout (Oppenheimer, 1973) that is associated with female selfies (7 out of 8 in this case) in particular (Burns, 2013) and three contained the $V$ sign that is common in Japanese selfies and is part of the kawaii juvenile cuteness style (Burdelski \& 
Mitsuhashi, 2010). Most selfies were of females (54\%; $25 \%$ were of males, $20 \%$ were mixed, and one was a posed selfie of a statue), agreeing with a previous international study of selfies in cities (Yazdani, 2014). Some of the (male and female) selfies had the purpose of sharing current clothes, hairstyles, or emotional states (e.g., sad). Two of the male selfies showed the effects of intoxication.

Screen grabs or screenshots from phones (Figure 5) are a feature of Twitter images is rarely discussed in the academic literature in the context of general social media image sharing (exception: Chen, Bentley, Holz, \& Xu, in press), although they have in other contexts (e.g., Brown, Chalmers, Bell, Hall, MacColl, \& Rudman, 2005; Shulgin, 1997-1998; Wigdor, Jiang, Forlines, Borkin, \& Shen, 2009). Video game screenshot sharing, for example, can "preserve and celebrate" gaming achievements that are essentially visual (Moore, 2014, p.145; see also: Poremba, 2007). Tweeting the current smartphone screen is a meformer strategy because it allows the user to share what they are doing, including applications such as chat systems (21\%), the music player (9\%), Facebook (5\%) and Twitter itself $(4 \%)$. Some uses of the screen grab have privacy implications because they allow the owner to convert private chat messages into a picture to share publicly.

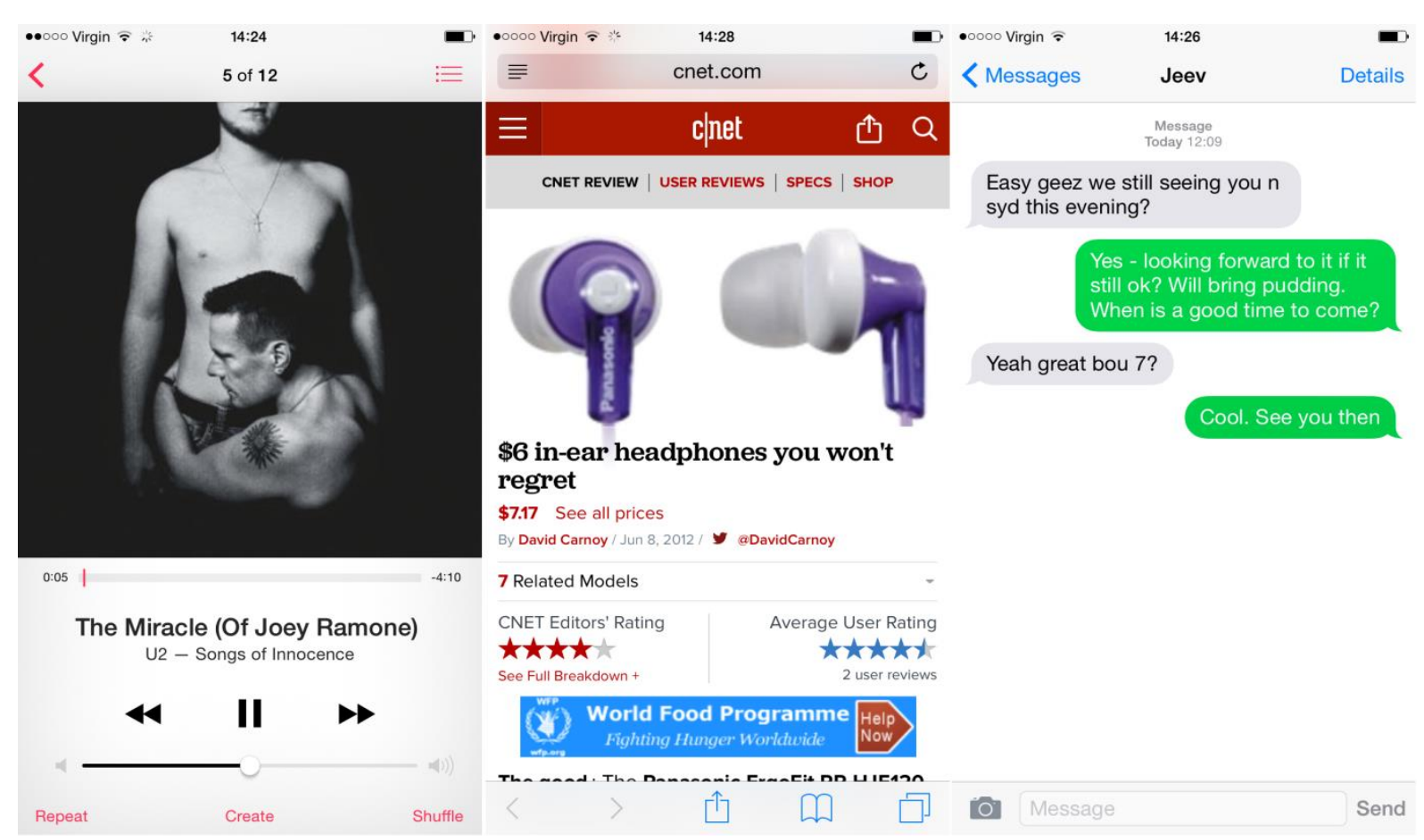

Figure 5. Three examples of screen grabs from a smartphone showing music playing on the device (left), a webpage currently open in the browser (middle) and a previously private text message chat (right). Images reproduced with permission of the participants.

About $9 \%$ of the images were simply pictures of text and about a third of the images included text that was important to the image. Half of the pictures of text were sentimental messages, sayings or advice (e.g., "People who walk away are not meant to be part of your future. Let them go. Your life is with those who stay, and they deserve all of you. - Bryant McGill"). These messages may have been more convenient to share in image form than in text form because the image naturally delineates the text of the saying from other tweets, giving them additional importance. The image format also allows more characters than a standard tweet, gives control over the spacing and font, and allows the user to comment on the message, for example by stating that they agree with it or can relate to it. Some other images had a similar purpose but included a patterned or related background and others 
included a picture of the famous person who composed the text as a background or at the side of the message. In addition to pictures dominated by text, other pictures had a balance between the text and the image. For example, some photographs with funny captions needed both the picture and the text to make sense. In other cases the image dominated the text but the text was still important. For example, one image was of a smiling nurse with small text advertising a nursing job. These images seem to fit an informer strategy because of often taking longer to make and giving a more general message.

The coding of the images into categories was complicated by some of them being layers of images, whether or not they were perceived as such by those that viewed them. For example, many of the screenshots embedded images, such as of artwork associated with music playing or photographs in webpages. There were also many photographs of images and other kinds of display, presumably due to the ease with which a smartphone photograph can be taken and shared. These included photographs of TV screens (e.g., sports results, soap events), signs (e.g., flight departure boards, pub signs), drawings, and homework.

Since Twitter is a real-time medium, images were classified as real-time unless there was evidence in them or in their associated tweets that they were not. This assumption will not always be correct, however, since the intended recipients of the image may have had access to other contextual information, such as previous tweets from the same sender, which would make it clear that an image was older. Moreover, it would not be possible in most cases to distinguish between an image sent within a few seconds of having been taken and an image sent later the same day, the next day, or weeks later (e.g., when back from holiday), when the person first had convenient access to an internet connection or a computer if they had had not tweeted from their phone.

Most of the tweets did not contain enough contextual information to suggest a specific purpose for sharing the image. Since most of the images seemed to be concurrent, they tended to share what the sender was doing, looking at or experiencing (i.e., the meformer strategy). The main purpose of the image sharing may therefore have been sharing of the personal experience, performed through relatively simple personal information exchanges with friends and acquaintances, as an extension of chatting with friends face-to-face or on the phone, which would agree with a previous study ( $\mathrm{Hu}$, Manikonda, \& Kambhampati, 2014). Images could have more specific motives in each case, however. For example, the photograph of a new pair of shoes could conceivably be a reminder to friends about an arranged night out (e.g., Harries, 2014) or fashion design coursework.

\section{Limitations}

An important limitation is that the sample from Twitter is not random but is pre-processed for sampling in an unknown way by Twitter. In addition, the use of Twitter is likely to change over time due to age shifts in its user base, competition from other services (e.g., Pinterest, Instagram), and new smartphones technologies. In addition, typical uses are likely to vary substantially over time according to the season. As a result of all these factors, it would not be reasonable to test for statistically significant differences between the UK and USA in the image categories. Although there are differences between the UK and USA distributions of categories, none seem large enough to be evidence of substantial underlying differences. This suggests that there are not major cultural differences but the results also underline the difficulty of making fair comparisons because of the extent to which cultural differences in life (e.g., ways of celebrating Christmas) overlap with differences in ways of using Twitter. 
Another limitation is that the nature of images shared in Twitter is partly the result of marketing decisions made by Twitter in terms of the services that they will allow to inject images into feeds. Nevertheless, some users have also hacked images from other platforms, such as Instagram, into Twitter (van Dijck, 2013; Bilton, 2012; Gordon, 2012).

\section{Conclusions}

Images shared on Twitter seem to be predominantly photographs used to update friends and acquaintances about what the user is currently doing, albeit within a relatively limited range of activity (not all activities get represented), and hence may be a visual extension of chatting face to face or on social media as a meformer strategy. These images seem to reflect fleeting moments during everyday life when a smartphone is available. Smartphone screenshot sharing allows people to broadcast non-photographic visual status updates as a relatively novel alternative method. The ease with which this is possible allows relatively complex images to form part of a casual chatting meformer pattern of use. Screengrab sharing has implications for privacy in some contexts, however, by allowing private text messages to be broadcast quickly.

Images are also used for wider information sharing, although this seems to be a minority activity. Images relating to news $(0.3 \%)$, politics $(0.4 \%)$ and sport $(2.4 \%)$ were rare in the set analysed (first author's post-hoc categorisation), but information sharing related to advertising $(7.8 \%)$, celebrities $(13.9 \%)$ or jokes $(4.3 \%)$ was more common. Text was important for about a third of the images, including photograph captions and picture backgrounds to sayings or famous quotes. Whilst this imagetext strategy is not new, its memetic variants are an enduring minority aspect of visual internet culture.

The results suggest several avenues for future research on social media images. First, it is important to follow up on the apparent use of images for chat or gossip. Previous research has suggested that the content of tweets can be partly implicit because of the limitations of the short text format (e.g., Thelwall, Buckley, \& Paltoglou, 2011) and a more in-depth study of tweets or interviews with users may shed more light on this issue. A more fine-grained analysis of the use of smartphone screenshots to communicate is also needed, given their relative novelty. It also important to connect and situate findings of imagesharing on Twitter to those on other platforms, such as Instagram, and identify similarities and differences in content, topical interests and users types (Ferrara, Interdonato, Tagarelli, 2014; Hu, Manikonda, Kambhampati, 2014). Another avenue for future research concerns the need for close reading of fleeting images in relation to broader digital cultural practices in an attempt to shed light on cross-platform genres and the degree to which Twitter images are unique social reflections on life or are firmly embedded within specific internet practices. Finally, it is important to investigate the privacy implications of the ease with which private information can be publicly broadcasted due to the multifunction nature of smartphones.

\section{References}

Alper, M. (2014). War on Instagram: Framing conflict photojournalism with mobile photography apps. New Media \& Society, 16(8), 1233-1248.

Alpers, S. (1983). The art of describing: Dutch art in the seventeenth century. London: Penguin Books.

Angus, E., Thelwall, M., \& Stuart D. (2008). General patterns of tag usage among university groups in Flickr. Online Information Review, 32(1), 89-101.

Bar-llan, J., Shoham, S., Idan, A., Miller, Y., \& Shachak, A. (2008). Structured versus unstructured tagging: A case study. Online Information Review, 32(5), 635-647. 
Batchen, G. (2008). Snapshots: Art history and the ethnographic turn. Photographies, 1(2), 121-142.

BBC (2014). Labour's Emily Thornberry quits over 'snobby' tweet http://www.bbc.co.uk/news/uk-politics-30139832

Beegan, G. (2008). The Mass Images: A Social History of Photomechanical Reproduction in Victorian London. New York: Palgrave.

Benjamin, W. (1936). The work of art in the age of mechanical reproduction. London: Penguin Books.

Berger, J. (2013). Understanding a photograph. London: Penguin Books.

Bilton, N. (2012). Twitter loses ability to properly display Instagram photos. 5 December, Bits Blog, New http://bits.blogs.nytimes.com/2012/12/05/twitter-loses-ability-to-properlydisplay-instagram-photos/ Accessed 21 February 2015.

Braden, S. (1983). Committing photography. London: Pluto Press.

Broersma, M., \& Graham, T. (2012). Social media as beat: tweets as a news source during the 2010 British and Dutch elections. Journalism Practice, 6(3), 403-419.

Bourlai, E., \& Herring, S.C. (2014). Multimodal communication on Tumblr: I have so many feels! In Proceedings of the 2014 ACM conference on Web science (pp. 171-175). New York: ACM Press.

Brown, A. (2013). The way she looked the day she died: Vernacular photography, memory, \& death. In Proceedings of the Art of Death and Dying Symposium held at the University of Houston (TX), October 25-27, 2012. https://journals.tdl.org/add/index.php/add/article/view/7043/6310

Brown, B., Chalmers, M., Bell, M., Hall, M., MacColl, I., \& Rudman, P. (2005). Sharing the square: collaborative leisure in the city streets. In ECSCW 2005 (pp. 427-447). Springer Netherlands.

Brubaker, J. R. (2008). Wants moar: Visual Media's use of text in LOLcats and silent film. Gnovis Journal, 8 (2), 117-124.

Burdelski, M., \& Mitsuhashi, K. (2010). "She thinks you're kawaii": Socializing affect, gender, and relationships in a Japanese preschool. Language in Society, 39(01), 65-93.

Burgess, J., Vis, F., \& Bruns, A. (2012). How many fake Sandy pictures were really shared on social media? 6 November. Guardian Data Blog. http://www.theguardian.com/news/datablog/2012/nov/06/fake-sandypictures-social-media Accessed 21 February 2015.

Burns, A. (2013). Discipline and the duckface: Social media, photography and perceptions of acceptable gender performance. Paper presented at 'Boys, Girls, Sex, Gender, and Everything Else', 1st March 2013, Loughborough University.

Cann, C. K. (2014). Tweeting death, posting photos, and pinning memorials: Remembering the dead in bits and pieces. In: C. M. Moreman, D. Lewis (eds) Digital Death: Mortality and Beyond in the Online Age. Santa Barbara, CA: Praeger (pp. 69-82).

Chalfen, R. (1987). Snapshot: Versions of life. http://muse.jhu.edu/books/9780879728748

Chen, Y. Y., Bentley, F., Holz, C., \& Xu, C. (in press). Sharing (and discussing) the moment: The conversations that occur around shared mobile media. In: Proceedings of Mobile $\mathrm{HCl} 2015$.

Chung, E. \& Yoon, J. (2013). An analysis of image use in Twitter message. Journal of the Korean Biblia Society for Library and Information Science, 24(4), 75-90.

Click, M. A., Lee, H., \& Holladay, H. W. (2013). Making Monsters: Lady Gaga, Fan Identification, and Social Media. Popular Music and Society, 36(3), 360-379.

Clifford, K. (2014). Amanda Knox: a picture of innocence. Celebrity Studies, 5(4), 504-507. [selfie with caption prominent at start of campaign: somo innocent] 
Cobley, P., \& Haeffner, N. (2009). Digital cameras and domestic photography: communication, agency and structure. Visual Communication, 8(2), 123-146.

Cohen, J. (1960). A coefficient of agreement for nominal scales. Educational and Psychological Measurement, 20 (1), 37-46.

Collings, B. (2014). \# selfiecontrol:@ CAZWELLnyc and the role of the ironic selfie in transmedia celebrity self-promotion. Celebrity Studies, 5 (4), 511-513.

De Choudhury, M., Diakopoulos, N., \& Naaman, M. (2012). Unfolding the event landscape on twitter: classification and exploration of user categories. In Proceedings of the ACM 2012 conference on Computer Supported Cooperative Work (pp. 241-244). New York: ACM Press.

Donath, J. (2007). Signals in social supernets. Journal of Computer-Mediated Communication, 13(1), 231-251.

Duggan, M. \& Smith, A. (2013). Social media update 2013. PewResearch Internet Project. http://www.pewinternet.org/2013/12/30/social-media-update-2013/

Dunbar, R. (1998). Grooming, gossip and the evolution of language. London: Faber and Faber Limited.

Durrant, A., Frohlich, D., Sellen, A., \& Uzzell, D. (2011). The secret life of teens: online versus offline photographic displays at home. Visual Studies, 26(2), 113-124.

Dutton, W. H., Blank, G., \& Groselj, D. (2013). Cultures of the internet: The internet in Britain. Oxford Internet Survey 2013 Report. http://oxis.oii.ox.ac.uk/wpcontent/uploads/2014/11/OxIS-2013.pdf

Ekman, M., \& Widholm, A. (2014). Twitter and the celebritisation of politics. Celebrity Studies, 5(4), 518-520.

Ensmenger, N. (2010). The computer boys take over. Cambridge: MIT Press.

Ferrara, E., Interdonato, R., \& Tagarelli, A. (2014). Online popularity and topical interests through the lens of Instagram. HT '14, September 1-4, Santiago, Chile. New York: ACM Press.

Fleiss, J.L. (1981). Statistical methods for rates and proportions (2nd ed.). New York: John Wiley.

Gomez Cruz, E. \& Ardévol, E. (2013). Performing photography practices in everyday life: Some ethnographic notes on a Flickr group. Photographies, 6(1), 35-44.

Gomez Cruz, E. \& Meyer, T. (2012). Creation and Control in the Photographic Process: iPhones and the Emerging Fifth Moment of Photography. Photographies, 5(2), 203 221.

Goriunova, O. (2012). Art platforms and cultural production on the internet. London: Routledge.

Goriunova, O. (2013). The force of digital aesthetics: on memes, hacking, and individuation. In Zeitschrift für Medienwissenschaft, 8, 70-87.

Goriunova, O. (2014). Fun and Software: Exploring Pleasure, Paradox and Pain in Computing. London: Bloomsbury.

Gupta, A., Lamba, H., Kumaraguru, P. (2013). \$1.00 per RT \#BostonMarathon \#PrayForBoston: Analyzing fake content on Twitter. eCrimes Researchers Summit (eCRS), 17-18 September, San Francisco, USA. IEEE, DOI: 10.1109/eCRS.2013.6805772

Gupta, A., Lamba, H., Kumaraguru, P., \& Joshi, A. (2013). Faking Sandy: Characterizing and identifying fake images on Twitter during Hurricane Sandy. In WWW 2013 Companion, May 13-17, Rio de Janeiro, Brazil. New York: AMC Press.

Hargittai, E., \& Litt, E. (2011). The tweet smell of celebrity success: Explaining variation in Twitter adoption among a diverse group of young adults. New Media \& Society, 13(5), 824-842. 
Harries, C. (2014). Ready for the weekend. On: Ready for the weekend [CD]. New York, NY:Columbia Records.

Holland, P. \& Spence, J. (eds) (1991). Family snaps: The meaning of domestic photography. London: Virago.

Hu, Y., Manikonda, L., \& Kambhampati, S. (2014). What we Instagram: A first analysis of Instagram photo content and user Types. In Proceedings of the Eight International AAAI Conference on Weblogs and Social Media.

Hunter, J. (1987). Image and word: The interaction of twentieth-century photographs and texts. Cambridge, MA: Harvard University Press.

Hutchins, B. (2011). The acceleration of media sport culture: Twitter, telepresence and online messaging. Information, Communication \& Society, 14(2), 237-257.

Ibrahim, Y. (2015). Instagramming life: Banal imaging and the poetics of the everyday. Journal of Media Practice, 16(1), 42-54.

Java, A., Song, X., Finin, T., \& Tseng, B. (2007). Why we twitter: Understanding microblogging usage and communities. In Proceedings of the 9th WebKDD and 1st SNA-KDD 2007 workshop on Web mining and social network analysis (pp. 56-65). New York: ACM Press.

Jörgensen, C. (1998). Attributes of images in describing tasks. Information Processing \& Management, 34(2), 161-174.

Jörgensen, C. (2003). Image Retrieval: Theory and Research. USA: Scarecrow Press.

Kassing, J. W., \& Sanderson, J. (2010). Fan-athlete interaction and Twitter tweeting through the Giro: A case study. International Journal of Sport Communication, 3(1), 113-128.

Klausen, J. (in press). Tweeting the jihad: Social media networks of Western foreign fighters in Syria and Iraq. Studies in Conflict \& Terrorism.

Landis, J.R. \& Koch, G.G. (1977). The measurement of observer agreement for categorical data. Biometrics, 33 (1), 159-174.

Larson, J. \& Sandbye, M. (eds) (2014). Digital Snaps: The New Face of Photography. London and New York: I. B. Taurus.

Leigh, C. (2009). Lurkers and Lolcats: An Easy Way From Out To In. Journal of Digital Research and Publishing, 2, 131-139.

Levy, Steven. (2001). Hackers. Heroes of the Computer Revolution. USA: Penguin Books. Marien, M. W. (2014). Photography: A cultural history (4 ed). London: Laurence King. Marlow, C., Naaman, M., Boyd, D. \& Davis, M. (2006). Position Paper, Tagging, Taxonomy, Flickr, Article, ToRead. Proceedings of the 15th International World Wide Web Conference, (Collaborative Web Tagging Workshop), May 22, Edinburgh, UK.

Marwick, A. \& boyd, d. (2011). To see and be seen: Celebrity practice on Twitter. Convergence: The International Journal of Research into New Media Technologies, 17(2), 139-158.

Miller, A. D., \& Edwards, W. K. (2007). Give and take: a study of consumer photo-sharing culture and practice. In Proceedings of the SIGCHI conference on Human factors in computing systems (pp. 347-356). New York: ACM Press.

Miltner, K. (2011). "Srsly Phenomenal: An Investigation into the Appeal of LOLCats". MSc Dissertation, London School of Economics, unpublished.

Mitchell, W. J. T. (2005). What do pictures want? The lives and loves of images. Chicago and London: The University of Chicago Press.

Moore, C. (2014). Screenshots as virtual photography: Cybernetics, remediation and affect. In: P. Arthur \& K. Bode (Eds.) Advancing digital humanities research, methods, theories. London: Palgrave Macmillan (pp. 258-273). 
Naaman, M., Boase, J., \& Lai, C. H. (2010). Is it really about me? Message content in social awareness streams. In Proceedings of the 2010 ACM conference on Computer supported cooperative work (pp. 189-192). New York: ACM Press.

Oppenheimer, J. R. (1973). Social and communicatory behavior in the Cebus monkeys. In Carpenter, C. R. (ed.), Behavioral Regulators of Behavior in Primates, Associated University Presses, Cranbury, NJ, pp. 251-257.

Panofsky, E. (1983). Meaning in the visual arts. Singapore: Peregrine Books.

Parmelee, J. H., \& Bichard, S. L. (2011). Politics and the Twitter revolution: How tweets influence the relationship between political leaders and the public. Lanham, MD: Lexington Books.

Poremba, C. (2007). Point and shoot remediating photography in gamespace. Games and Culture, 2(1), 49-58.

Rainie, L., Brenner, J. \& Purcell, K. (2012). Photos and Videos as Social Currency Online. PewResearch Internet Project. http://www.pewinternet.org/2012/09/13/photos-andvideos-as-social-currency-online/

Reading, A. (2011). The London bombings: Mobile witnessing, mortal bodies and globital time. Memory Studies, 4(3), 298-311.

Robinson, M. \& Picard, D. (eds) (2009). The Framed World: Tourism, Tourists and Photography. Farnham and Burlington: Ashgate.

Rogers, S. (2014). What fuels a Tweet's engagement? March 10. https://blog.twitter.com/2014/what-fuels-a-tweets-engagement Accessed 21 February 2015.

Rose, G. (2010). Doing Family Photography: The Domestic, The Public and The Politics of Sentiment. Aldershot: Ashgate.

Rose, G. (2012). Visual methodologies: An introduction to researching with visual materials. London: Sage.

Rubinstein D. and Sluis K. (2013) The digital image in photographic culture; The algorithmic image and the crisis of representation. In: The Photographic Image in Digital Culture, ed. Martin Lister, London: Routledge.

Sanderson, J., \& Cheong, P. H. (2010). Tweeting prayers and communicating grief over Michael Jackson online. Bulletin of science, technology \& society, 30(5), 328-340.

Sarvas, R. \& Frohlich, D. M. (2011). From Snapshots to Social Media - The Changing Picture of Domestic Photography. London: Springer.

Shatford, S. (1986). Analyzing the subject of a picture: A theoretical approach. Classification Quarterly. 6(33), 39-62.

Shatford, S. (1994). Some Issues in the Indexing of Images. Journal of the American Society for Information Science, 45(8), 583-588.

Shifman, L. (2013). Memes in digital culture. Cambridge, MA: MIT Press.

Shulgin, A. (1997-1998). Desktop Is. Art project. http://www.easylife.org/desktop/

Sontag, S. (1978). On Photography. London: Penguin Books.

Taylor, C. (2011). Twitter Unveils Photo \& Video Sharing. MashableUK. 1 June. http://mashable.com/2011/06/01/twitter-photos/ Accessed 21 February 2015.

ten Bhömer, M., Helmes, J., O'Hara, K., \& van den Hoven, E. (2010). 4Photos: a collaborative photo sharing experience. In Proceedings of the 6th Nordic Conference on HumanComputer Interaction: Extending Boundaries (pp. 52-61). New York: ACM Press.

Thelwall, M., Buckley, K., \& Paltoglou, G. (2011). Sentiment in Twitter events. Journal of the American Society for Information Science and Technology, 62(2), 406-418. 
Thelwall, M., Wouters, P., \& Fry, J. (2008). Information-Centred Research for large-scale analysis of new information sources, Journal of the American Society for Information Science and Technology, 59(9), 1523-1527.

Thoring, A. (2011). Corporate tweeting: analysing the use of Twitter as a marketing tool by UK trade publishers. Publishing research quarterly, 27(2), 141-158.

Tufekci, Z. (2008). Grooming, gossip, Facebook and MySpace: What can we learn about these sites from those who won't assimilate? Information, Communication \& Society, $11(4), 544-564$.

Twitter Blog. (2011a). Share a photo via text message. 21 September. https://blog.twitter.com/2011/share-photo-text-message Accessed 21 February 2015.

Twitter Blog. (2011b). Twitter and iOS 5: Sharing made simple. 12 October. https://blog.twitter.com/2011/twitter-and-ios-5-sharing-made-simple Accessed 21 February 2015.

Twitter Help Center (no date). Posting photos on Twitter. https://support.twitter.com/articles/20156423-posting-photos-on-twitter Accessed 21 February 2015.

Urry, J., \& Larsen, J. (2011). The tourist gaze 3.0. London: Sage.

Van Dijck, J. (2008). Digital photography: communication, identity, memory. Visual Communication, 7(1), 57-76.

Van Dijck, J (2013). The culture of connectivity: A critical history of social media. Oxford and New York: Oxford University Press.

Van House, N., Davis, M., Ames, M., Finn, M., \& Viswanathan, V. (2005). The uses of personal networked digital imaging: an empirical study of cameraphone photos and sharing. In $\mathrm{CHI} 05$ extended abstracts on Human factors in computing systems ( $\mathrm{pp}$. 1853-1856). New York: ACM Press.

Van House, N.A. (2011). Personal photography, digital technologies, and the uses of the visual. Visual Studies, 25(1), pp.125-134.

Van Straten, R. (1994). An Introduction to Iconography. New York: Taylor and Francis.

Vis, F., Faulkner, S., Parry, K., Manyukhina, Y. \& Evans, L. (2013) Twitpic-ing the Riots:

Analysing Images Shared on Twitter during the 2011 U.K. Riots. Twitter and Society. New York: Peter Lang.

Weltevrede, E., Helmond, A., \& Gerlitz, C. (2014). The politics of real-time: A device perspective on social media platforms and search engines. Theory, Culture \& Society, 31(6), 125-150.

Wiggins, B.E., \& Bowers, B.G. (2014). Memes as genre: A structurational analysis of the memescape. New Media \& Society. Online first. DOI: $10.1177 / 1561444814535194$.

Whitson, G. (2012). Instagram Broke the Ability to Share Photos on Twitter, Here's How to Fix It. Lifehacker. http://lifehacker.com/5966050/instagram-broke-the-abilityto-share-photos-on-twitter-heres-how-to-fix-it Accessed 21 February 2015

Wigdor, D., Jiang, H., Forlines, C., Borkin, M., \& Shen, C. (2009). WeSpace: the design development and deployment of a walk-up and share multi-surface visual collaboration system. In Proceedings of the SIGCHI Conference on Human Factors in Computing Systems (pp. 1237-1246). New York: ACM Press.

Wood, N. T., \& Burkhalter, J. N. (2014). Tweet this, not that: A comparison between brand promotions in microblogging environments using celebrity and companygenerated tweets. Journal of Marketing Communications, 20(1-2), 129-146. 
Wu, S., Hofman, J. M., Mason, W. A., \& Watts, D. J. (2011). Who says what to whom on Twitter. In Proceedings of the 20th international conference on World wide web (pp. 705-714). New York: ACM Press.

Yazdani, M. (2014). Gender, age, and ambiguity of selfies on Instagram. http://lab.softwarestudies.com/2014/02/gender-age-and-ambiguity-of-selfieson.html

Zhao, X., Salehi, N., Naranjit, S., Alwaalan, S., Voida, S., \& Cosley, D. (2013). The many faces of Facebook: Experiencing social media as performance, exhibition, and personal archive. In Proceedings of the SIGCHI Conference on Human Factors in Computing Systems (pp. 1-10). New York: ACM Press. 